\title{
Role of Aspirin for primary prevention of cardiovascular and all-cause mortality events in diabetes: An updated meta-analysis of randomized controlled trials.
}

\author{
Hua $\mathrm{Ma}^{1}$, QIng $\mathrm{Gu}^{2}$, Huining $\mathrm{Niu}^{1}$, Xiaohua $\mathrm{Li}^{1}$, and Rong Wang ${ }^{3}$ \\ ${ }^{1}$ Xiangyang Central Hospital \\ ${ }^{2}$ Peking Union Medical College Hospital \\ ${ }^{3}$ The Second People's Hospital of Kunshan City
}

May 31, 2021

\begin{abstract}
Background: The use of Aspirin in the primary prevention of cardiovascular disease (CVD) is still a topic of debate, especially in patients with diabetes. The present meta-analysis aims to rule out the efficacy of Aspirin in patients with diabetes and to compare the effectiveness of Aspirin with a placebo (or no treatment) for the primary prevention of CVD and all-cause mortality events in people with diabetes. Materials and Methods: An extensive and systematic search was conducted in Medline (via PubMed), Cinahl (via Ebsco), Scopus, and Web of Sciences from 1988 to December 2020. A detailed literature search was conducted using Aspirin, cardiovascular disease (CVD), diabetes, and efficacy to identify trials of patients with diabetes who received Aspirin for primary prevention of CVD. Demographic details with the primary outcome of events and bleeding outcomes were analyzed. The risk of bias (RoB) in included studies was evaluated using the QUADAS-2 tool. Results: A total of 5 studies out of 13 were included with 23,570 diabetic patients; 11,738 allocated to Aspirin and 11,832 allocated to the placebo group. In patients with diabetes, there was no difference between Aspirin and placebo with respect to the risk of all-cause death with a confidence interval (CI) varying 0.63 to 1.17 . In addition, there were no differences in the bleeding outcomes with an odds ratio of 1.4411 (CI 0.47 to 4.34). Conclusion: Aspirin has no significant risk on primary endpoints of cardiovascular events and the bleeding outcomes in diabetic patients compared to placebo. More research on the use of Aspirin alone or in combination with other antiplatelet drugs is required in patients with diabetes to supplement currently available research.
\end{abstract}

\section{Introduction}

Cardiovascular disease (CVD) is one of the primary causes of mortality in people with diabetes, accounting for $>70 \%$ of deaths in these people. ${ }^{1}$ Low-dose aspirin is considered the most widely used agent for preventing cardiovascular disease..$^{2-4}$, and its use in subjects with diabetes is also recommended by various guidelines ${ }^{5-6}$. Several primary prevention aspirin trials, mostly done before 2000 , suggested a reduction in myocardial infarction and stroke, although not mortality ${ }^{7}$ but at the cost of increased bleeding events. ${ }^{8-10}$ However, the role of Aspirin in the primary prevention of CVD is still unclear and under debate. According to guidelines by the American Diabetes Association, the American Heart Association, and the American College of Cardiology Foundation advocate using low-dose Aspirin for the primary prevention of CVD in adults with diabetes, but which should be based on the individual risk of CVD and risk of bleeding ${ }^{11}$. Aim: The present meta-analysis was done to rule out the role of Aspirin in patients with diabetes and to compare the effectiveness of Aspirin with a placebo (or no treatment) for the primary prevention of CVD and all-cause mortality events in people with diabetes.

\section{Materials and Methods}


We followed the STARD statement (Standards for Reporting of Diagnostic Accuracy Studies) normative recommendations in this study with the registration number XU\#/IRB/2020/1005.

\section{Data sources, eligibility, and Data extraction}

We searched in Medline (via PubMed), Cinahl (via Ebsco), Scopus, and Web of Sciences until December 2020 using the predefined search terms aspirin, cardiovascular disease (CVD), diabetes, efficacy, OR meta-analysis until December 2020. Ten trials included in a previous meta-analysis; ${ }^{12}$ were also identified and included in the present meta-analysis. The inclusion criteria include only randomized clinical trials of Aspirin, except one which was a cross-sectional study. The Aspirin was compared with placebo or no treatment in patients with or without diabetes, and without a history or clinical evidence of CVD; screened for the eligibility. Studies had to be controlled (placebo or control group) but could be open-label or blinded. The patients above 18 years of age either who have diabetes was included, and full text was acquired for further evaluation if the citation was deemed pertinent. Studies were excluded if the patient was below 18 years of age, nonrandomized studies comparing Aspirin with another antiplatelet agent, or use of Aspirin in combination with any other antiplatelet drug compared with placebo.

The data extracted from the selected trials included appropriate study-level information on type of study design; baseline of the study period, source of participants; total population including proportion of men; location; age range at baseline; allocation concealment; blinding; intervention and dosage; medication compliance; duration of the therapy, completeness of follow-up and also included trial population with diabetes as described in Table 1 .

The quality of risk involved was assessed using the QUADAS-2 (Quality Assessment of Diagnostic Accuracy Studies 2) criterion. If any disagreement arose, it was resolved by consensus among reviewers ${ }^{24}$.

The outcomes of major adverse cardiovascular event/s [MACE; defined as a combination of nonfatal myocardial infarction (MI), nonfatal stroke and cardiovascular death], other cardiovascular outcomes, all-cause mortality, and adverse events; and risk estimates were also evaluated in diabetic patients to rule out the efficacy of Aspirin to placebo.

\section{Statistical Analysis}

The meta-analysis was carried out using NCSS Software. The odds ratio for all-cause death, CVS-caused death, MI, and stroke was evaluated in patients taking Aspirin compared to placebo in diabetes. The random-effects model was used with statistical significance at a p-value less than 0.05 . The odds ratio of various bleeding events was also done to do the forest plot of studies analyzing the effect of Aspirin compared to placebo in diabetic patients. The logarithm of the measures was used to construct the forest plot. Again, the random-effects model was used with statistical significance at a p-value less than 0.05 .

\section{Results}

\section{Literature Search Results}

Table 1 summarizes the key demographic characteristics of all trials previously done between the period of 1988 to 2020, including patients with diabetes. Five trials were eligible out of 13 trials which included only patients with diabetes for the analysis to rule out the efficacy of Aspirin for cardiovascular events. Table 2 summarizes the trials investigating the effect of Aspirin in the primary prevention of cardiovascular events and its risk of bleeding outcomes in individuals only with diabetes.

The present Meta-analysis comprised 23,570 diabetic patients; 11,738 allocated to Aspirin and 11,832 allocated to the control or placebo group.

Risk of Bias Assessment

According to the QUADAS-2 tool, all of the included experiments had a low chance of bias (Table 3).

Meta-analysis Results: 
Figure 1 and Table 4 show the forest plot of the odds ratio of all-cause death compared to Aspirin with placebo in diabetic patients using the Random Effects Model. Figure 1 is statistically significant with a p-value less than 0.05 under the random-effects model with CI varying from 0.63 to 1.17. The confidence intervals slightly crossed the line of no effect showing an insignificant combined result with an average odds ratio of 0.86 for all-cause death among patients taking Aspirin compared to placebo. Figure 2 and Table 5 showed the forest plot of the odds ratio of CVS-related death compared to Aspirin with placebo using Random Effects Model. The result is statistically significant with a p-value less than 0.05 under the randomeffects model with CI varying from 0.61 to 1.63 . The confidence intervals slightly crossed the line of no effect, showing an insignificant combined result with an average odds ratio of 1.0032 for CVS-related deaths among patients taking Aspirin compared to placebo. Figure 3 and Table 6 show the forest plot and results of the odds ratio for the risk of MI compared to Aspirin with placebo using Random Effects Model. The result is statistically insignificant with a p-value of more than 0.05 under the random-effects model with CI varying from 0.80 to 1.03 . The confidence intervals slightly crossed the line of no effect showing an insignificant combined result with an average odds ratio of 0.9106 for MI risk among patients taking Aspirin compared to placebo. Figure 4 and Table 7 show the forest plot and results of the odds ratio for stroke risk compared to Aspirin with placebo in diabetic patients using Random Effects Model. The given figure is statistically insignificant with a p-value more than 0.05 under the random-effects model with CI varying from 0.79 to 1.09. The confidence intervals slightly cross the line of no effect showing an insignificant combined result with an average odds ratio of 0.9328 for stroke risk among patients taking Aspirin compared to placebo. Figure 5 and Table 8 show the forest plot and result of the odds ratio for the risk of bleeding in diabetic patients compared to Aspirin with placebo using the Random Effects Model. The given figure is statistically insignificant with a p-value more than 0.05 under the random-effects model with CI varying from 0.47 to 4.34. The confidence intervals crossing the line of no effect showed an insignificant combined result with an average odds ratio of 1.4411 for bleeding events among diabetic patients taking Aspirin when compared to placebo.

\section{Discussion}

Myocardial infarction (MI) and ischemic stroke are the leading causes of mortality and morbidity in patients with type 2 diabetes. ${ }^{18}$ The strength of the evidence for the use of antiplatelet agents as secondary prevention ${ }^{19-22}$ has led to the suggestion that Aspirin might be helpful for primary prevention of cardiovascular events in diabetes.

In order to evaluate the role of Aspirin in the prevention of cardiovascular events, especially in diabetic patients, the primitive search of the present meta-analysis was compared to its risk of bleeding. Similarly, to compare the benefits over the harms of Aspirin, the chances of risk of all-cause death, CVS-related death, MI, stroke, and risk of bleeding were compared between the patients taking Aspirin and no aspirin group (placebo).

When the odds ratio of all-cause death was compared with Aspirin plus placebo in diabetic patients, the confidence intervals slightly crossed the line of no effect, which shows an average odds ratio of 0.86 . It signifies that the $\mathrm{p}>0.05$ has no specific effect of Aspirin on all-cause death in diabetes. The average odds ratio of CVS-related death compared to Aspirin with placebo in diabetic patients was insignificant with a value of 1.0; CI varied from 0.61 to 1.63, showing no significant role of Aspirin in CVS-related death. The chances of the risk of MI in aspirin patients were also insignificant compared with placebo with a confidence interval of 0.9 , slightly crossing the line of no effect. The possibility of a chance of stroke in the aspirin group was also insignificant, with a p-value of 0.93 , resulting in no specific risk of Aspirin for stroke in diabetic patients. There were no differences in the rates of bleeding outcomes between patients who received Aspirin and those who received a placebo.

All included trials were consistent in reporting that no significant effect of Aspirin on primary prevention of CV events in individuals with diabetes, which is similar to the present meta-analysis except for the JPAD trial $^{15}$; which significantly reported lower risk in the aspirin group when compared with placebo showing an inefficacy of low dose of Aspirin. A follow-up study of 10 years was performed for the same trial, which 
together constitutes the JPAD2 study ${ }^{23}$ which concluded that a low dose of Aspirin has no role in the risk of $\mathrm{CV}$ events but included an increased risk of gastrointestinal bleeding. A recent trial by ASCEND group ${ }^{17}$ only showed the beneficial effect of Aspirin in preventing serious vascular events in diabetic patients with no cardiovascular events.

Several Meta-analyses and randomized trials have been previously conducted to rule out the role of Aspirin in the primary prevention of cardiovascular events, including diabetic patients. However, due to the low ratio of diabetic patients in the studies as mentioned in Table 1, these studies were excluded in the present meta-analysis to reduce the chances of risk of bias as there was the inability to perceive event rate data specifically for patients with diabetes.

\section{Conclusion}

The role of Aspirin did not reduce the risk of cardiovascular events and has been considered with low risk for primary prevention of cardiovascular events. However, the present meta-analysis reported no significant risk of Aspirin in the primary prevention of cardiovascular events when compared with placebo in diabetic patients. In addition, we found no significant risk of Aspirin on the primary endpoints of cardiovascular events and cardiovascular mortality over the risk of bleeding outcomes in diabetic patients. However, further investigations of Aspirin combined with other antiplatelet drugs can be conducted to rule out the possible advantages in preventing cardiovascular risk among patients of diabetes.

\section{Declarations:}

Ethics approval and consent to participate

All procedures performed in studies involving human participants were in accordance with the ethical standards of the institutional and/or national research committee of the Xianyang Central Hospital and Peking Union Medical College Hospital. And comply with the 1964 Helsinki Declaration and its later amendments or comparable ethical standards. The written informed consent was taken from all the patients.

Consent to publish: Not Applicable

Availability of data and materials: The datasets used and/or analyzed during the current study are available from the corresponding author on reasonable request.

Competing interests: The authors declare that they have no competing interests

Funding: No Funding was received.

Authors' Contributions: HM: has designed the concept; QG, Data acquisition; HN drafted the manuscript; XLliterature search; RW Final proof reading and editing. All Authors read and approved the manuscript before submission.

Acknowledgements: None Declared.

\section{REFERENCES}

1. Bulugahapitiya U, Siyambalapitiya S, Sithole J, Idris I. Is diabetes a coronary risk equivalent? Systematic review and meta-analysis.Diabet Med 2009; 26: 142-148.

2. Hennekens CH, Dyken ML, Fuster V. Aspirin as a therapeutic agent in cardiovascular disease: a statement for healthcare professionals from the American Heart Association. Circulation 1997; 96:2751-3.

3. Ajani UA, Ford ES, Greenland KJ, Giles WH, Mokdad AH. Aspirin use among U.S. adults: Behavioral Risk Factor Surveillance System. AmJ Prev Med 2006; 30: 74-7.

4. Ittaman SV, VanWormer JJ, Rezkalla SH. The role of Aspirin in the prevention of cardiovascular disease. Clin Med Res.2014; 12: 147-54.

5. U.S. Preventive Services Task Force. Aspirin for the primary prevention of cardiovascular events: recommendation and rationale. Ann Intern Med 2002; 136:157-60. 
6. Colwell JA, American Diabetes Association. Aspirin therapy in diabetes. Diabetes Care 2003; 26:S87S88.

7. Ridker PM, Cook NR, Lee I-M, et al. A randomized trial of low-dose Aspirin in the primary prevention of cardiovascular disease in women. $N$ Engl J Med 2005; 352: 1293-304.

8. Steering Committee of the Physicians' Health Study Research Group. Final report on the aspirin component of the ongoing Physicians' Health Study. N Engl J Med 1989; 321: 129-35.

9. The Medical Research Council's General Practice Research Framework. Thrombosis prevention trial: randomised trial of low-intensity oral anticoagulation with warfarin and low-dose Aspirin in the primary prevention of ischaemic heart disease in men at increased risk.Lancet 1998; 351: 233-41.

10. Hansson L, Zanchetti A, Carruthers SG, et al. Effects of intensive blood-pressure lowering and lowdose Aspirin in patients with hypertension: principal results of the Hypertension Optimal Treatment (HOT) randomised trial. Lancet 1998; 351: 1755-62.

11. Pignone M, Alberts MJ, Colwell JA, Cushman M, Inzucchi SE, Mukherjee D et al. Aspirin for primary prevention of cardiovascular events in people with diabetes. J Am Coll Cardiol 2010; 55: 2878-2886.

12. Kunutsor SK, Seidu S, Khunti K. Aspirin for primary prevention of cardiovascular and all-cause mortality events in diabetes: updated meta-analysis of randomized controlled trials. Diabet Med. 2017;34(3):316-27.

13. Aspirin effects on mortality and morbidity in patients with diabetes mellitus. Early Treatment Diabetic Retinopathy Study report 14. ETDRS Investigators. JAMA 1992; 268: 1292-1300.

14. Belch J, MacCuish A, Campbell I, Cobbe S, Taylor R, Prescott R et al. The prevention of progression of arterial disease and diabetes (POPADAD) trial: factorial randomised placebo-controlled trial of Aspirin and antioxidants in patients with diabetes and asymptomatic peripheral arterial disease. $B M J$ 2008; 337: $a 1840$.

15. Ogawa H, Nakayama M, Morimoto T, Uemura S, Kanauchi M, Doi N et al. Low-dose aspirin for primary prevention of atherosclerotic events in patients with type 2 diabetes: a randomized controlled trial.JAMA 2008; 300: 2134-2141.

16. Sasso F C, Marfella R * Pagano A, Porta G, Signoriello G et al. Lack of effect of Aspirin in primary CV prevention in type 2 diabetic patients with nephropathy: results from 8 years follow-up of NID-2 study. Acta Diabetol. 2014

17. Group ASC, Bowman L, Mafham M, Wallendszus K, Stevens W, Buck G, et al. Effects of Aspirin for primary prevention in persons with diabetes mellitus. N Engl J Med. 2018;379(16):1529-39.

18. Fox CS, Coady S, Sorlie PD, et al. Increasing cardiovascular disease burden due to diabetes mellitus: the Framingham Heart Study. Circulation . 2007; 115 (12):1544-1550.

19. Tierney, Fennessy F,Hayes DB.ABC of arterial and vascular disease: secondary prevention of peripheral vascular disease. BMJ 2000;320:1262-5.

20. Belch JJ, Topol EJ, Agnelli G, Bertrand M, Califf RM, Clement DL, et al. Critical issues in peripheral arterial disease detection and management. A call to action. Arch Intern Med 2003;163:884-92.

21. Scottish Intercollegiate Guidelines Network. Diagnosis and management of peripheral arterial disease: a national clinical guideline. 2006. No 89. www.sign.ac.uk/pdf/sign89.pdf.

22. Norgren L, Hiatt WR, Dormandy J, Mehler MR,Harris KH, Fowkes FGR, et al. Inter-society consensus for the management of peripheral arterial disease (TASC II). Eur J Vasc Endovasc Surg.2007;33(suppl 1):S1-75.

23. Yoshihiko Saito Y, Okada S, Ogawa H, Soejima H, Sakuma M et al. Low-Dose Aspirin for Primary Prevention of Cardiovascular Events in Patients With Type 2 Diabetes Mellitus. 10-Year Follow-Up of a Randomized Controlled Trial. Circulation. 2017;135:659-670.

24. Whiting PF, Rutjes AW, Westwood ME, et al; QUADAS-2 Group. QUADAS-2: a revised tool for the quality assessment of diagnostic accuracy studies. Ann Intern Med. 2011;155(8):529-536

Figure Legends

Figure 1: Forest plot of Odds Ratio of All-Cause Death in comparison of Aspirin with Placebo in diabetes 
Figure 2: Forest plot of Odds Ratio of CVS related Death in comparison of Aspirin with Placebo in diabetes

Figure 3: Forest plot of Odds Ratio of Odds Ratio of risk of MI in comparison of Aspirin with Placebo in diabetes

Figure 4: Forest plot of odds ratio for the risk of stroke in comparison of Aspirin with placebo in diabetic patients

Figure 5: Forest plot of Odds Ratio of Odds Ratio of risk of bleeding in comparison of Aspirin with Placebo in diabetes

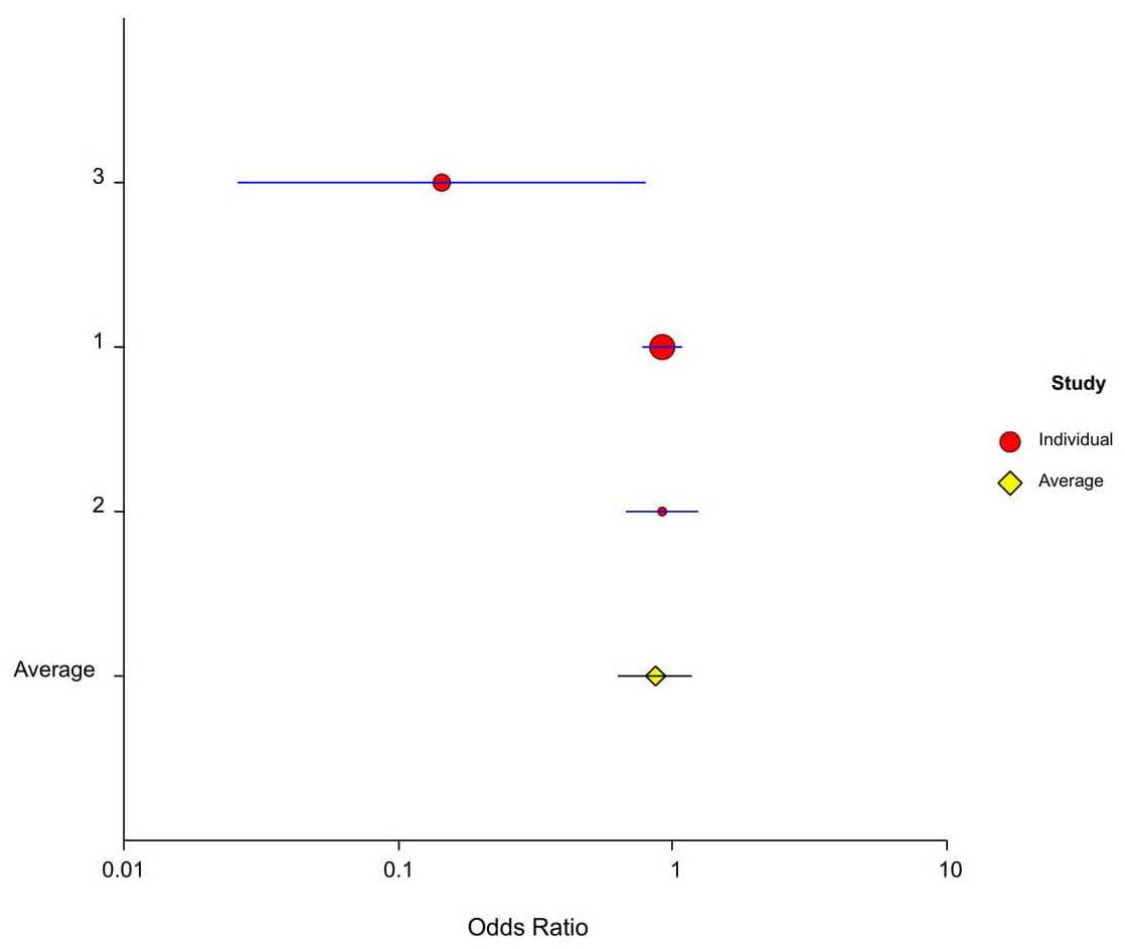




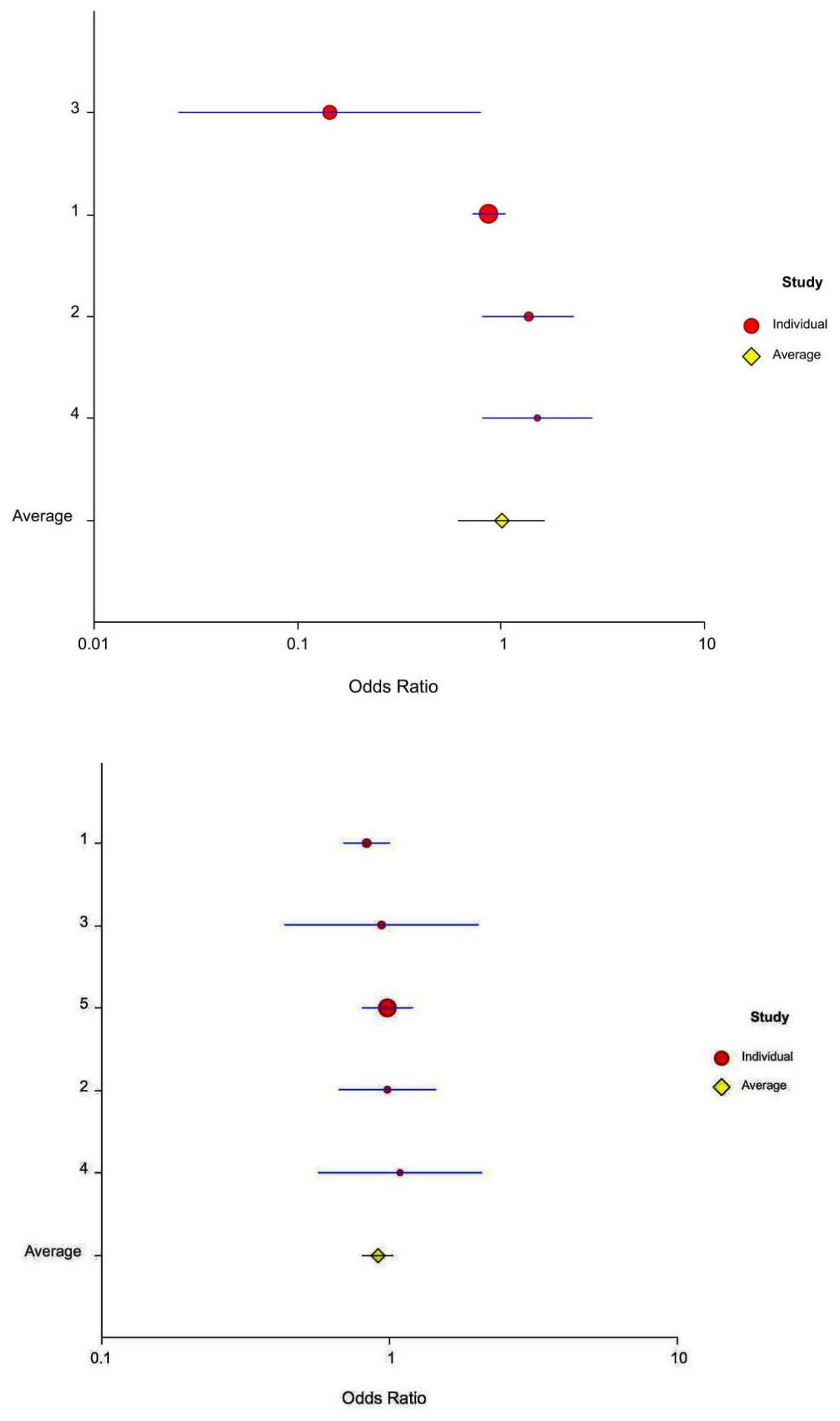



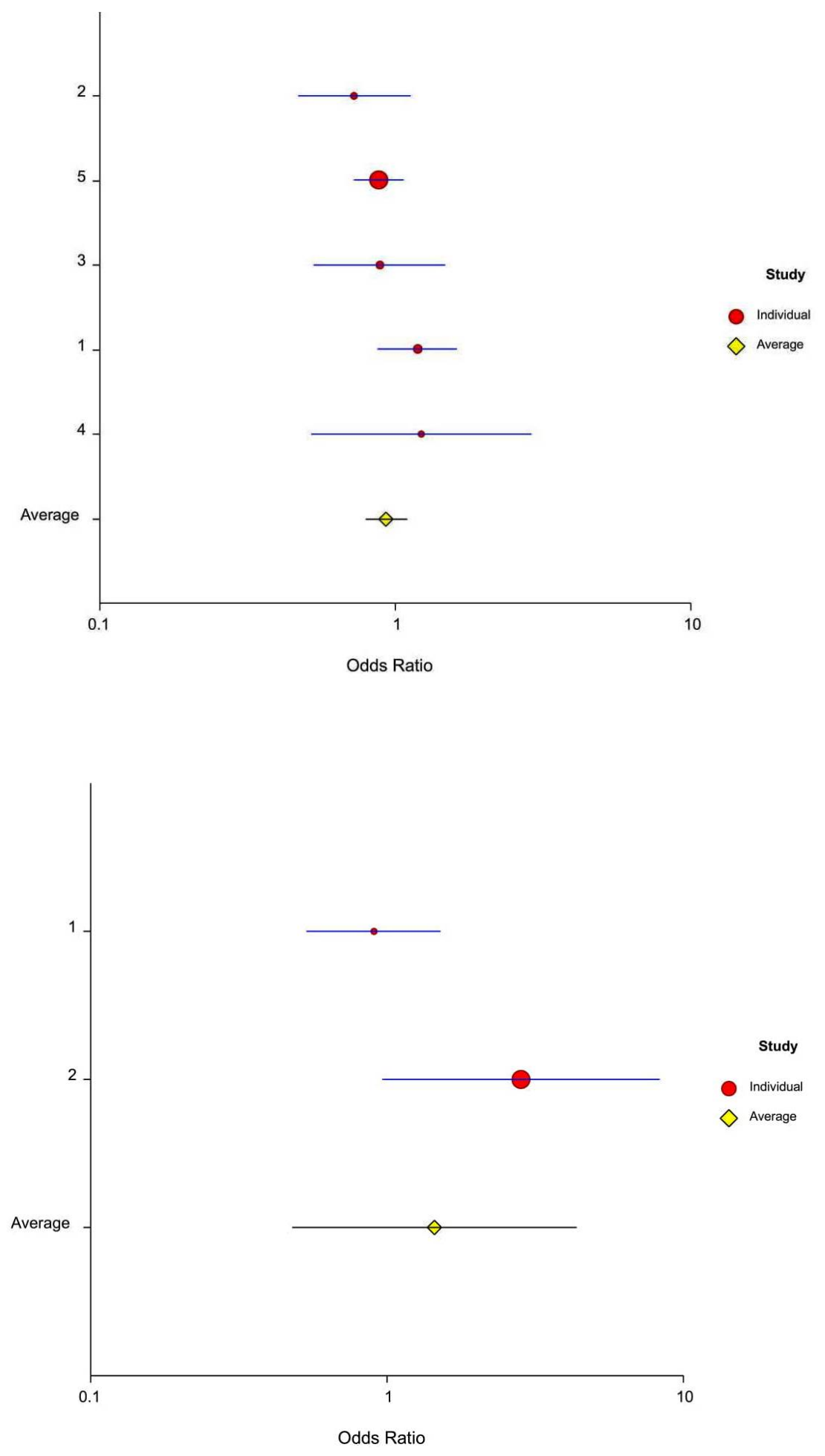

\section{Hosted file}

Tables New for Aspirin.docx available at https://authorea.com/users/417037/articles/524287role-of-aspirin-for-primary-prevention-of-cardiovascular-and-all-cause-mortality-events- 
in-diabetes-an-updated-meta-analysis-of-randomized-controlled-trials 